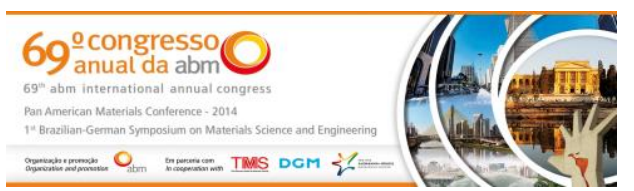

Tema: Materiais cerâmicos, compósitos e poliméricos

\title{
PET PROCESSADO NA PRESENÇA DE EXTENSOR DE CADEIA: ESTIMATIVA DA MASSA MOLAR POR MEIO DAS MEDIDAS DE TORQUE EM MISTURADOR INTERNO DE LABORATÓRIO*
}

Albaniza Alves Tavares ${ }^{1}$

Diêgo Fabrício Alcântara Silva²

Daniela de Lourdes Anjos Coutinho Simões Andrade 3

Eduardo Luís Canedo 4

Suédina Maria de Lima Silva ${ }^{5}$

\section{Resumo}

No presente trabalho, poli (tereftalato de etileno) virgem (PETV) e pós-consumo (PETPC) foram aditivados com um oligômero multifuncional estirênico-acrílico-epóxi (Joncryl POLYAD PR 002) comercializado como extensor de cadeia para polímeros de condensação como o PET, visando avaliar o comportamento da massa molar destes polímeros durante o processamento. PETV e PETPC, com e sem a incorporação do aditivo, foram preparados em misturador interno Haake Rheomix, com rotores tipo roller, empregando diferentes níveis do extensor de cadeia. As massas molares foram estimadas a partir de medições de torque e temperatura. Os resultados mostram que as estimativas com base em medições de torque em tempo real foi um indicador rápido e preciso da eficácia da extensão da cadeia do PET. O aditivo extensor de cadeia compensou a perda de massa molar durante 0 processamento, o efeito foi afetado pelo nível de aditivo presente.

Palavras-chave: PET; Massa molar; Extensor de cadeia.

\section{PET PROCESSED IN THE PRESENCE OF EXTENDER CHAIN: ESTIMATE OF MOLAR MASS THROUGH THE TORQUE MEASURES IN INTERNAL MIXER OF LABORATORY}

\section{Abstract}

In this study, poly (ethylene terephthalate) virgin (PETV) and post-consumer (PETPC) were additives with a styrenic-acrylic-epoxy multifunctional oligomer (Joncryl POLYAD PR 002) marketed as chain extender for condensation polymers such as PET to evaluate the behavior of molar mass these polymers during processing. PETV and PETPC, with and without the incorporation of additive, were prepared in an internal mixer Haake Rheomix with roller type rotors, using different levels of the chain extender. The molar masses were estimated from measurements of torque and temperature. The results show that the estimates based on torque measurements in real time was a fast and accurate indicator of the effectiveness of the extension of the PET chain. The lost of molar mass was offset by the chain extender additive, the effect was affected by the level of additive.

Keywords: PET; Molar mass; Extender chain.

1 Pós-Graduanda, Programa de Pós Graduação em Ciências e Engenharia de Materiais, UFCG, Campina Grande, PB, Brasil.

2 Graduando, Departamento de Engenharia de Materiais, UFCG, Campina Grande, PB, Brasil.

3 Eng ${ }^{a}$ de Materiais, Doutora, Professora Departamento de Engenharia de Materiais, UFCG, Campina Grande, PB, Brasil.

4 Eng.Químico, Doutor, Pesquisador do Instituto de Tecnologia de Pernambuco, ITEP, Recife, PE, Brasil.

5 Enga de Materiais, Doutora, Professora Departamento de Engenharia de Materiais, UFCG, Campina Grande, PB, Brasil.

\footnotetext{
* Contribuição técnica ao $69^{\circ}$ Congresso Anual da ABM - Internacional e ao 14ํㅡㄹ ENEMET - Encontro Nacional de Estudantes de Engenharia Metalúrgica, de Materiais e de Minas, 21 a 25 de julho de 2014, São Paulo, SP, Brasil.
} 


\section{INTRODUÇÃO}

O poli (tereftalato de etileno) (PET) é um poliéster empregado em larga escala por indústrias de todo mundo [1]. Por apresentar uma excelente relação entre propriedades mecânicas, térmicas, de barreira e baixo custo de produção, este polímero é bastante empregado na fabricação de uma grande variedade de produtos de embalagens para envase de bebidas como refrigerantes, águas, sucos e óleos comestíveis, além de uma vasta gama de artigos injetados, termoformados e extrusados [2-8].

Por se tratar de um polímero muito aplicado em produtos com um tempo de vida útil curto e por ter como característica a capacidade de ser reciclado várias vezes, o PET ocupa uma posição de destaque no mercado de resinas recicláveis [9-12]. A reciclagem contribui com a redução do descarte irracional e inadequado de plásticos pós- consumo além de diminuir o consumo de matéria prima primária [13].

Durante seu ciclo de vida e processo de reciclagem, o PET pode sofrer degradação mecânica, hidrolítica, térmica e oxidativa, limitando seu uso em muitas aplicações devido à redução da sua massa molar, por conseguinte, da sua viscosidade, da resistência do fundido e de propriedades mecânicas [14-16]. Uma maneira de minimizar a perda de massa molar é através da aditivação do PET com extensores de cadeia, os quais possuem sítios ativos que reagem com os grupos terminais das cadeias desses polímeros de condensação, constituindo assim uma nova cadeia polimérica estendida [17-20].

Em geral os extensores de cadeia para o PET são compostos do tipo bis-anidridos $[4,17,21]$, bis-oxazolinas [22,23], fosfatos [24] diissocianatos [14,25] e bis-epóxidos [18,26-28], que quando empregados em teores adequados possibilitam o aumento da massa molar e consequentemente da viscosidade do PET durante 0 processamento por fusão $[17,19,29]$. A extensão de cadeia e o consequente aumento na massa molar possibilitam ampliar a aplicação do PET pós-consumo reciclado.

Existe uma vasta gama de extensores de cadeia disponíveis comercialmente para o PET dentre os quais podemos citar: o dianidrido piromelítico (PMDA), o bisoxazolina fenileno (PBO), o trifenil fosfito (TPP). Extensores de cadeia bifuncionais promovem a extensão da cadeia linear, enquanto os extensores de cadeia de tri e tetrafuncionais promovem a ramificação de cadeia [19].

Dependendo da natureza, da reatividade e número de grupos funcionais presentes na molécula do extensor de cadeia, diferentes estruturas macromoleculares podem ser obtidas, conduzindo a diferentes propriedades do material resultante. Além disso, variáveis tais como concentração do extensor de cadeia e condições de processamento empregadas durante a reação de extensão da cadeia do PET pode influenciar no peso molecular do polímero e, por conseguinte, na sua viscosidade, na resistência do fundido e propriedades mecânicas.

Este trabalho foi realizado com o objetivo de avaliar a influência de um oligômero multifuncional de estireno-acrílico-epóxi (Joncryl - POLYAD PR 002), comercializado como extensor de cadeia para polímeros de condensação como o poli (tereftalato de etileno) (PET), no aumento da massa molar e, consequentemente, no controle da degradação do PET. O efeito do teor do extensor de cadeia foi avaliado. Objetivouse também estimar a massa molar do PET durante o processamento (determinação online) a partir de dados de torque obtidos do misturador interno.

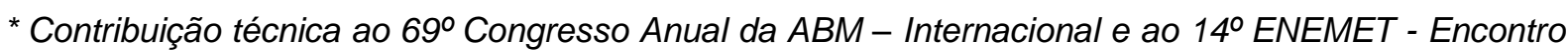
Nacional de Estudantes de Engenharia Metalúrgica, de Materiais e de Minas, 21 a 25 de julho de 2014, São Paulo, SP, Brasil.
} 


\section{MATERIAIS E MÉTODOS}

\subsection{Materiais}

Neste estudo foram utilizados dois tipos de poli(tereftalato de etileno) - PET: PET pós-consumo (PETPC), fornecido pela empresa DEPET/PB/Brasil na forma de flocos, proveniente de embalagens de refrigerante (garrafas) incolor e o PET virgem (PETV), grau garrafa, de nome comercial Cleartuf $\AA$ Turbo ${ }^{\mathrm{TM}}$ fornecido pelo Grupo Mossi e Ghisolfi (M\&G)/PE/Brasil na forma de grânulos.

O aditivo POLYAD PR 002 (blenda composta pelos aditivos Joncryl 4368 e Joncryl 4370) fornecido pela BASF, na forma de grânulos, foi empregado como extensor de cadeia para os polímeros PETV e PETPC.

\subsection{Métodos}

Os polímeros (PETV e PETPC) foram secos, antes da etapa de processamento, em estufa a vácuo na temperatura de $160^{\circ} \mathrm{C}$ por um período de $6 \mathrm{~h}$, permanecendo sob vácuo por mais $16 \mathrm{~h}$ a $30^{\circ} \mathrm{C}$. Este procedimento foi necessário em função da alta higroscopicidade do PET. Em seguida, amostras de PETV e PETPC, sem e com o extensor de cadeia (POLYAD PR 002), foram preparadas em um misturador interno acoplado ao reômetro de torque Haake Rheomix 3000QC da PolyLab QC, utilizando rotores do tipo roller operando a $265^{\circ} \mathrm{C}$ por $16 \mathrm{~min}$ e velocidade de $60 \mathrm{rpm}$. Foi avaliado o comportamento da massa molar do PET em presença de três concentrações de extensor de cadeia $(1,5 \%, 3 \%$ e $5 \%$ em massa) a partir de medições de torque e temperatura obtidas pelo equipamento acima citado.

A massa dos insumos foi calculada de forma a se obter um fator de enchimento $(f)$ de $85 \%$ de acordo com a Equação 1 [30], onde $\rho$ corresponde à densidade da mistura na temperatura de processamento e $V_{F}$ ao volume livre da câmara de processamento do misturador interno.

\section{RESULTADOS E DISCUSSÃO}

$$
m=\rho f V_{F}
$$

O PET virgem (PETV) e o PET pós-consumo (PETPC), sem a incorporação do aditivo extensor de cadeia, foram processados em triplicata a fim de avaliar a reprodutibilidade dos dados. Os resultados das curvas de torque e temperatura em função do tempo de processamento (t) estão apresentados nas Figuras 1 e 2, respectivamente.

A alimentação do PETV e do PETPC foi realizada intermitentemente, em função do grande volume gerado ao qual preenchia rapidamente a câmara do misturador e impedia a alimentação em uma única etapa. É possível observar aumento nos valores de torque com a alimentação, devido ao atrito entre as partículas e a deformação plástica, seguida da diminuição gradativa (a partir de $t=3,0 \mathrm{~min}$ ). $\mathrm{Na}$ medida em que o polímero funde este atua como lubrificante, consequentemente 0 atrito e a deformação são reduzidos até a completa fusão do material com os valores de torque se aproximando de zero para o tempo total de processamento (16 min).

O PETV apresentou uma variação de torque entre as amostras por isso em média podemos considerar que a alimentação deste elevou o torque até um valor máximo de aproximadamente $166 \mathrm{Nm}$ (Figura 1a) e depois de $\mathrm{t}=2,5 \mathrm{~min}$ este valor foi

\footnotetext{
* Contribuição técnica ao $69^{\circ}$ Congresso Anual da ABM - Internacional e ao 14ํㅡㄹ ENEMET - Encontro Nacional de Estudantes de Engenharia Metalúrgica, de Materiais e de Minas, 21 a 25 de julho de 2014, São Paulo, SP, Brasil.
} 


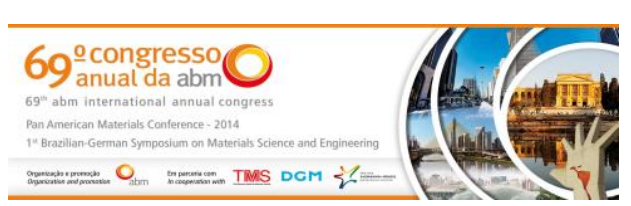

reduzido para aproximadamente $24 \mathrm{Nm}$ com a fusão total deste polímero. Após este tempo o torque foi diminuindo gradativamente até um valor de aproximadamente $3 \mathrm{Nm}$ para o tempo total de processamento empregado neste estudo. O menor valor de torque apresentado pelo PETPC quando comparado ao PETV, pode estar relacionado com a origem do PETPC, pois por tratar-se de um insumo oriundo de produtos pós-consumo possivelmente apresenta certo nível de degradação e consequentemente cadeias de tamanhos menores.

Com relação a temperatura do fundido (Figura 2), observa-se inicialmente uma diminuição da mesma, atribuída às trocas de calor com o ambiente ocorrido durante a abertura da câmara para alimentação do polímero e a absorção de calor pelo polímero durante a sua fusão. Em seguida, a temperatura aumenta e atinge um platô em aproximadamente 9 min tanto para o PETV quanto para o PETPC.

De uma forma geral, observa-se uma boa reprodutibilidade dos dados, sendo a diferença entre as curvas de torque e temperatura muito pequenas, para ambos os PET's, virgem (PETV) e pós-consumo (PETPC), realizados em triplicata. Resultado análogo foi obtido por Duarte (2013) em estudo semelhante [31].
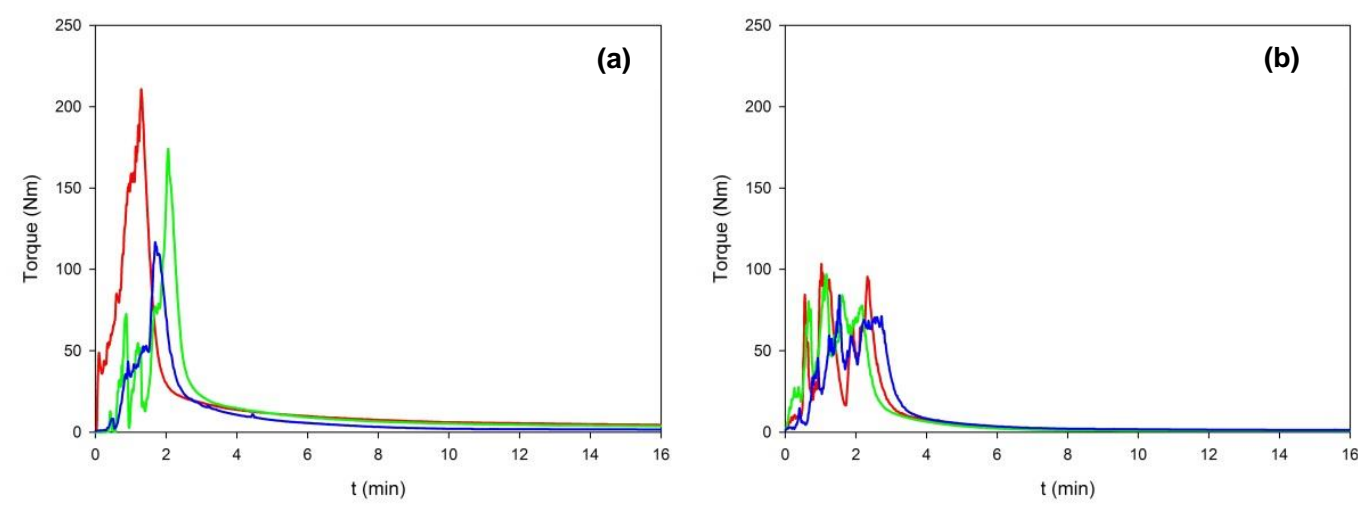

Figura 1. Torque versus tempo para o PETV (a) e o PETPC (b).
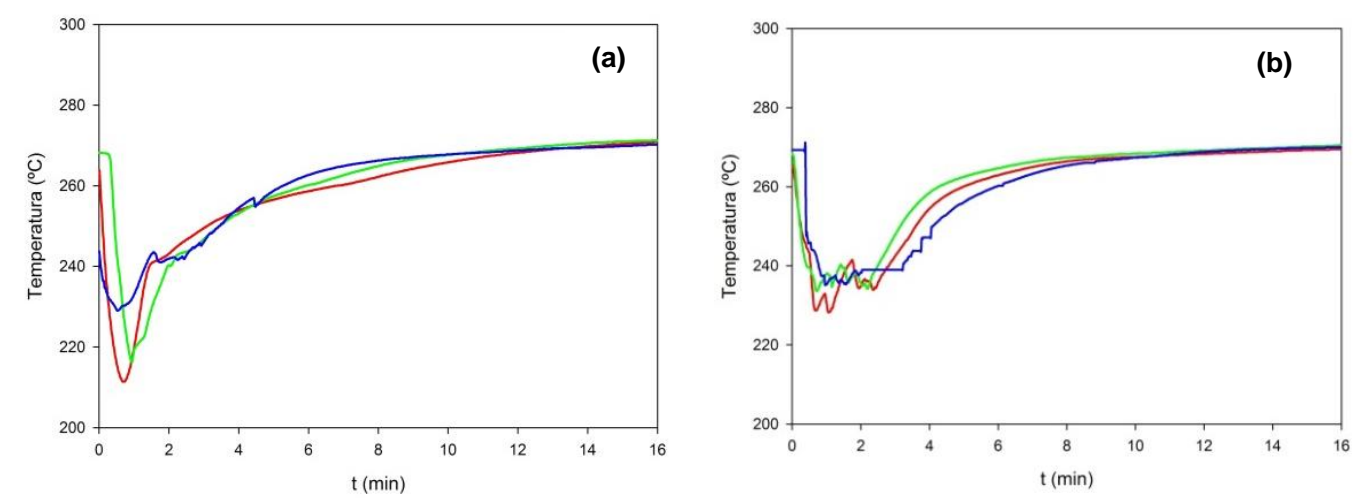

Figura 2. Temperatura versus tempo para o PETV (a) e o PETPC (b).

A influência do teor de extensor de cadeia $(1,5,3,0$ e 5,0\%) nos valores de torque (Figura 3) e temperatura do fundido (Figura 4) do PETV e PETPC foi avaliada.

É possível observar aumentos nos valores de torque no início do processo, quando o material, que se encontra no estado sólido, está sendo alimentado seguido da diminuição deste em função da fusão do polímero que leva consequentemente à redução da viscosidade. A abertura da câmara de processamento, depois de

\footnotetext{
* Contribuição técnica ao $69^{\circ}$ Congresso Anual da ABM - Internacional e ao 14ํㅡㄹ ENEMET - Encontro Nacional de Estudantes de Engenharia Metalúrgica, de Materiais e de Minas, 21 a 25 de julho de 2014, São Paulo, SP, Brasil.
} 

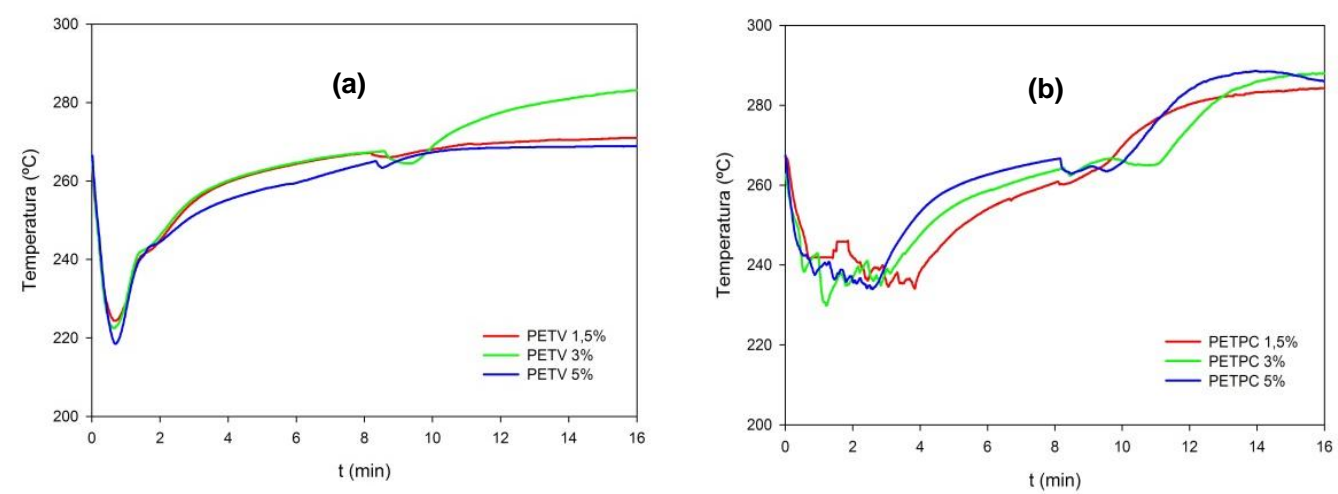

Figura 4. Temperatura versus tempo para o PETV (a) e o PETPC (b) aditivados com $1,5 \%, 3 \%$ e $5 \%$ de extensor de cadeia.

De forma geral, o aumento do torque e da temperatura com a adição de 1,5\%, 3\% e $5 \%$ do aditivo extensor de cadeia podem estar associados a aumentos na viscosidade e consequentemente nas massas molares do PET. A Figura 5 mostra imagens do PETV e do PETPC processados na presença do extensor de cadeia onde é possível verificar o aumento na viscosidade destes polímeros quando processados na presença do mesmo, sendo o aumento proporcional ao teor de extensor empregado. Resultados semelhantes foram observados por Duarte [31] empregando o mesmo extensor e Villalobos e colaboradores com o Joncryl 4368, o qual compõe juntamente com o Joncryl 4370 o aditivo POLYAD PR 002 utilizado neste estudo [18].

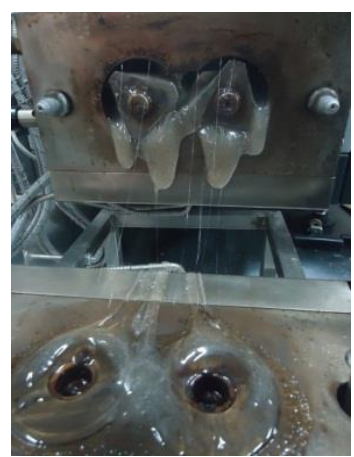

PETV

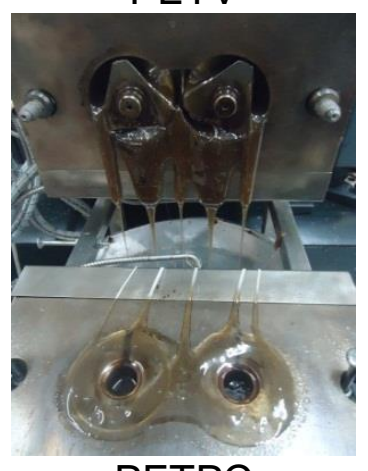

PETPC

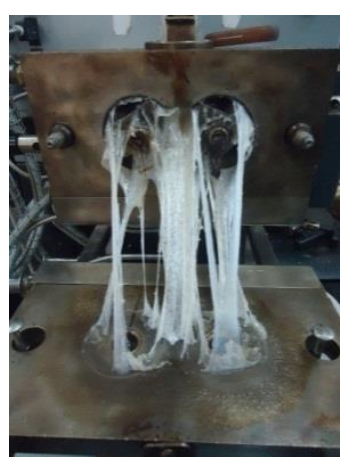

PETV1,5\%

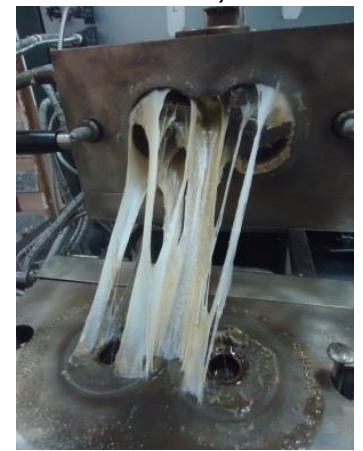

PETPC1,5\%

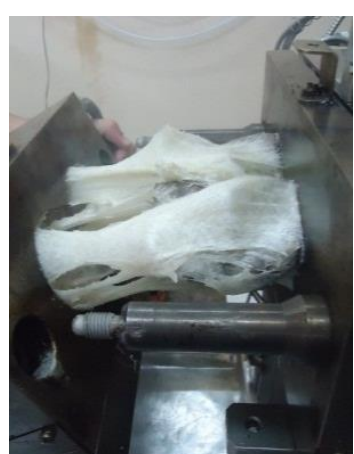

PETV3\%

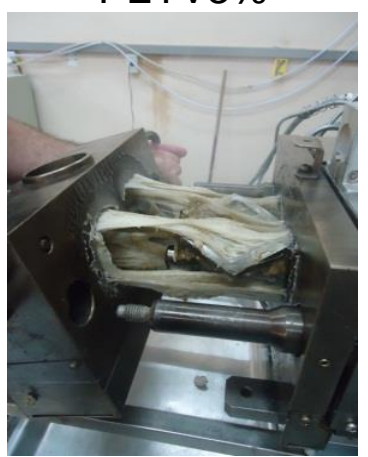

PETPC3\%

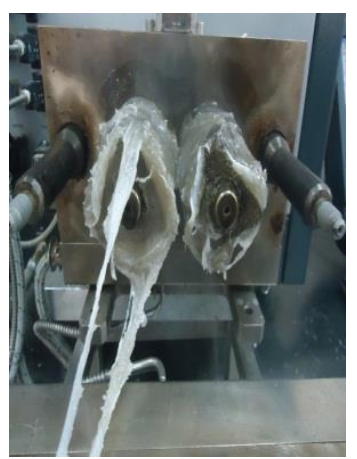

PETV5\%

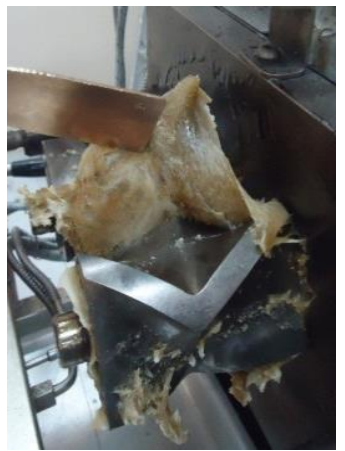

PETPC5\%

Figura 5. Imagens do PETV e do PETPC processados na ausência e na presença de 1,5\%; 3\% e 5\% do extensor de cadeia.

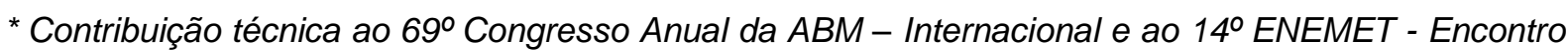
Nacional de Estudantes de Engenharia Metalúrgica, de Materiais e de Minas, 21 a 25 de julho de 2014, São Paulo, SP, Brasil. 


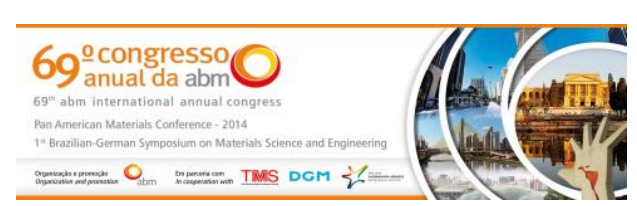

A fim de avaliar o efeito real do teor de extensor de cadeia na massa molar do PET foi feita uma estimativa desta para o PET virgem (PETV) e o PET pós-consumo (PETPC) sem e com o aditivo extensor de cadeia a partir dos dados numéricos fornecidos pelo equipamento (misturador interno) para dois intervalos de tempo: entre 3 e 7 min (antes da incorporação do aditivo) e entre 10 e 16 min (depois da incorporação do aditivo). O parâmetro $\alpha$ foi estimado a partir da Equação 2 e permitiu quantificar o aumento relativo da massa molar média do PET após a incorporação do aditivo [32].

$$
\alpha=\left(\frac{M_{w 2}}{M_{w 1}}\right)^{\frac{1}{3.5}}
$$

Nessa equação o valor $\alpha=3.5$ é uma constante reológica usada para a maioria dos polímeros semi-cristalinos [32], $M_{w 1}$ e $M_{w 2}$ são as massas molares antes e depois da incorporação do aditivo, respectivamente. Os resultados obtidos estão apresentados nas Tabelas 1 e 2. Nota-se que os valores dos parâmetros $\alpha$ apresentados pelo PETV e pelo PETPC foram alterados pela incorporação do aditivo. Maiores teores de aditivo resultaram em maiores valores do parâmetro $\alpha$ indicando maiores aumento da massa molar.

Tabela 1. Resultados experimentais para o PETV

\begin{tabular}{cccc}
\hline Amostra & $M_{w 1}$ & $M_{w 2}$ & $\alpha$ \\
\hline PETV & 8,62 & $-45,27$ & 1,60 \\
PETV 1,5\% & $5,79 \times 10^{-3}$ & 0,0289 & 1,58 \\
PETV 3\% & $2,52 \times 10^{-3}$ & 0,0253 & 1,93 \\
PETV 5\% & $5,51 \times 10^{-4}$ & 0,0616 & 3,85 \\
\hline
\end{tabular}

Tabela 2. Resultados experimentais para o PETPC

\begin{tabular}{cccc}
\hline Amostra & $M_{w 1}$ & $M_{w 2}$ & $\alpha$ \\
\hline PETPC & $6,09 \times 10^{-4}$ & $3,78 \times 10^{-3}$ & 1,68 \\
PETPC 1,5\% & $2,81 \times 10^{-3}$ & $-0,57$ & 4,56 \\
PETPC 3\% & $2,67 \times 10^{-3}$ & 0,0408 & 2,18 \\
PETPC 5\% & $3,7 \times 10^{-4}$ & $-1,78$ & 11,27 \\
\hline
\end{tabular}

A Figura 6 mostra como o teor de aditivo (1,5\%, 3\% e 5\%) influenciou na massa molar do PETV e do PETPC. As massas molares apresentadas pelo PETV e pelo PETPC não aditivados foram semelhantes. A adição de 1,5\% do extensor de cadeia ao PETV estabilizou o mesmo, isto é, o nível de extensão promovida pelo aditivo apenas compensou a perda na massa molar sofrida e o acréscimo de $3 \%$ elevou em aproximadamente $21 \%$. Por outro lado, a adição de $5 \%$ do aditivo resultou em um aumento significativo na massa molar. Os resultados sugerem a necessidade de adição de um teor mínimo do aditivo (nesse caso 3,0\%) para promover um incremento na massa molar do PET virgem (PETV). Para o PET pós-consumo (PETPC) a adição de $1,5 \%$ elevou a massa molar em 2,5 vezes quando comparado a PETPC não aditivado e para $5 \%$ esse aumento foi da ordem de aproximadamente 6,5 vezes. Entretanto, a adição de $3 \%$ do extensor apenas compensou a perda de massa molar. Mais investigações estão sendo conduzidas para se ter um melhor entendimento sobre o aumento da massa molar do PETPC processado na presença de $3 \%$ do extensor de cadeia e os resultados obtidos serão apresentados em outra publicação.

\footnotetext{
* Contribuição técnica ao $69^{\circ}$ Congresso Anual da ABM - Internacional e ao 14ํㅡㄹ ENEMET - Encontro Nacional de Estudantes de Engenharia Metalúrgica, de Materiais e de Minas, 21 a 25 de julho de 2014, São Paulo, SP, Brasil.
} 

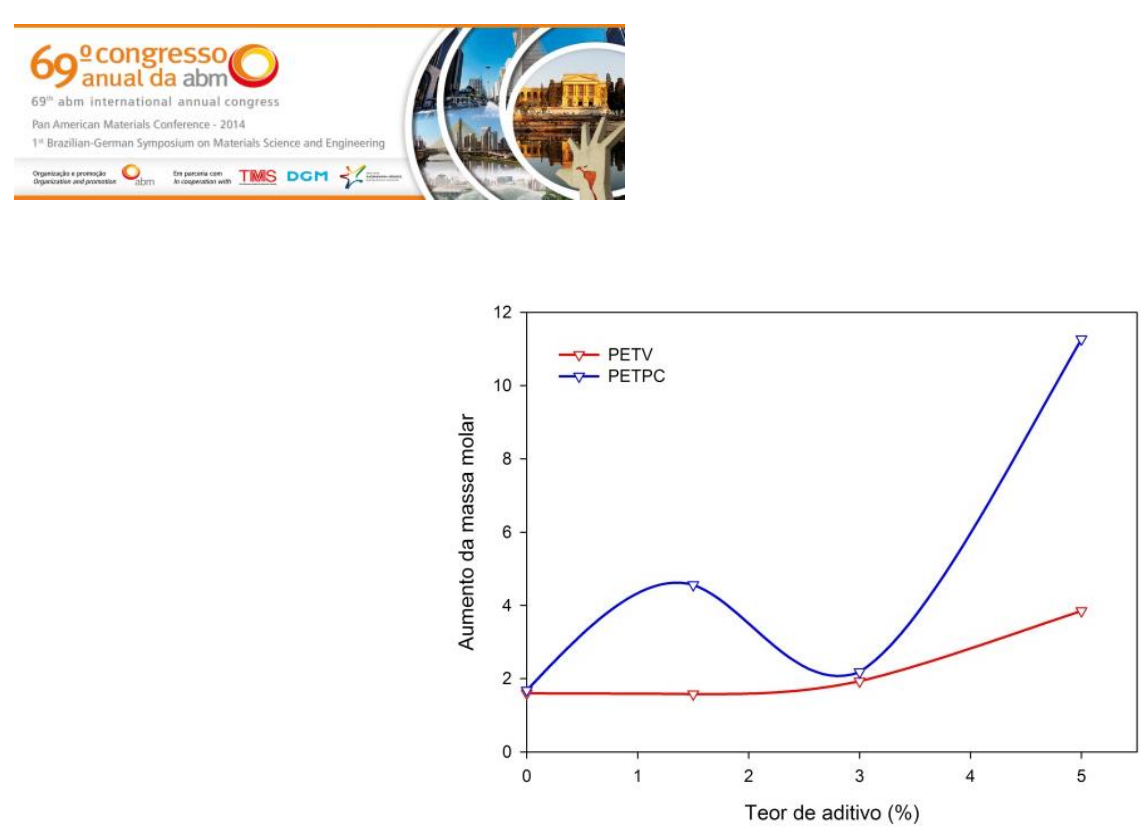

Figura 6. Massa molar em função do teor do aditivo extensor de cadeia para o PETV e PETPC.

\section{CONCLUSÃO}

Os resultados indicam que o extensor de cadeia usado neste estudo foi eficiente em promover o controle da degradação do PET (PETV e PETPC) durante o processamento, conforme indicado pelos aumentos da viscosidade e da massa molar dos mesmos, sendo os valores obtidos função do teor de extensor de cadeia empregado. Tais resultados mostram também que a estimativa da massa molar do PET durante o processamento (determinação online) a partir das curvas obtidas diretamente do misturador interno é uma ferramenta rápida e precisa para avaliar a eficiência da extensão de cadeia do PET.

\section{Agradecimentos}

Os autores agradecem a Capes e ao CNPq pelo apoio financeiro a M\&G, a Basf e a DEPET pela doação do PET virgem, do aditivo extensor de cadeia e do PET pósconsumo, respectivamente.

\section{REFERÊNCIAS}

1 Massey S, Cloutier P, Sanche L, Roy D. Mass spectrometry investigation of the degradation of polyethylene terephtalate induced by low-energy $(<100 \mathrm{eV})$ electrons. Radiation Physics and Chemistry. 2008; 77: 889-897.

2 Zhang Y, Guo W, Zhang H, Wu C. Influence of chain extension on the compatibilization and properties of recycled poly(ethylene terephthalate)/linear low density polyethylene blends. Polymer Degradation and Stability. 2009; 94: 1135-1141.

3 Raffa P, Coltelli M-B, Savi S, Bianchi S, Castelvetro V. Chain extension and branching of poly(ethylene terephthalate) (PET) with di- and multifunctional epoxy or isocyanate additives: An experimental and modelling study. Reactive and Functional Polymers. 2012; 72: 50-60.

4 Awaja F, Pavel D. Recycling of PET. European Polymer Journal. 2005; 41: 1453-1477.

5 Chilton T, Burnley S, Nesaratnam S. A life cycle assessment of the closed-loop recycling and thermal recovery of post-consumer PET. Resources, Conservation and Recycling. 2010; 54: 1241-1249.

6 Dimitrov N, Kratofil Krehula L, Ptiček Siročić A, Hrnjak-Murgić Z. Analysis of recycled PET bottles products by pyrolysis-gas chromatography. Polymer Degradation and Stability. 2013; 98: 972-979.

\footnotetext{
* Contribuição técnica ao $69^{\circ}$ Congresso Anual da ABM - Internacional e ao 14ํㅡㄹ ENEMET - Encontro Nacional de Estudantes de Engenharia Metalúrgica, de Materiais e de Minas, 21 a 25 de julho de 2014, São Paulo, SP, Brasil.
} 
26 Bikiaris DN, Karayannidis GP. Chain extension of polyesters PET and PBT with two new diimidodiepoxides. II. Journal of Polymer Science Part A: Polymer Chemistry. 1996; 34: 1337-1342.

27 Bikiaris DN, Karayannidis GP. Thermomechanical analysis of chain-extended PET and PBT. Journal of applied polymer science. 1996; 60: 55-61.

28 Japon S, Boogh L, Leterrier Y, Månson JAE. Reactive processing of poly(ethylene terephthalate) modified with multifunctional epoxy-based additives. Polymer. 2000; 41: 5809-5818.

29 Incarnato L, Scarfato P, Di Maio L, Acierno D. Structure and rheology of recycled PET modified by reactive extrusion. Polymer. 2000; 41: 6825-6831.

30 Canedo LE. Notas de Aula Mini Curso Processamento no Misturador Interno. Campina Grande, Universidade Federal de Campina Grande. 2013.

31 Duarte, IS. Uso de extensor de cadeia visando a modificação da estrutura molecular do PET. 2013. 56f (Mestrado). Dissertação (Mestrado em Ciência e Engenharia de Materiais), Universidade Federal de Campina Grande, Campina Grande, PB.

32 Gregory, DR. Rheological properties of molten poly(ethylene terephthalate). Journal of Applied Polymer Science.1972; 16: 1479-1487.

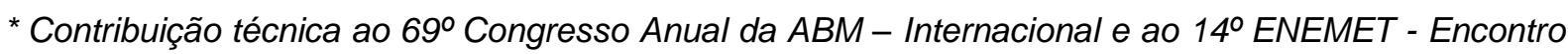
Nacional de Estudantes de Engenharia Metalúrgica, de Materiais e de Minas, 21 a 25 de julho de 2014, São Paulo, SP, Brasil. 